\title{
Improved time response for polymer fibre Bragg grating based humidity sensors
}

\author{
W. Zhang* , D. J. Webb \\ Photonics Research Group, Aston University, Birmingham, B4 7ET, UK, \\ G.-D. Peng \\ School of Electrical Engineering and Telecommunications, University of New South Wales, Sydney, \\ NSW 2052, Australia
}

\begin{abstract}
In this work we experimentally investigate the response time of humidity sensors based on polymer optical fibre (POF) Bragg gratings. By the use of etching with acetone we can control the diameter of POF based on poly (methyl methacrylate) in order to reduce the diffusion time of water into the polymer and hence speed up the relative wavelength change caused by humidity variations. A much improved response time of 11 minutes has been achieved by using a POF FBG with a reduced diameter of 135 microns.
\end{abstract}

Keywords: Polymer optical fibre, fibre Bragg grating, PMMA, humidity, response time

\section{INTRODUCTION}

Polymer optical fibre (POF) has been available for several decades. Its use has been overshadowed in the last two decades by the success of silica optical fibre, however it is widely used in short-haul communication links due to its high propagation loss. Although recent technological advances have helped promote POF as a lower cost alternative to glass fibre or copper at short distances and bit rates of up to $10 \mathrm{Gbps}$, the physical and chemical properties of polymeric materials are rather different to silica, potentially making it attractive for researchers to exploit in device and sensing applications. More recently, fibre Bragg gratings (FBG) have been inscribed into step index ${ }^{[1]}$ and microstructured ${ }^{[2]}$ POF. POF is often based on poly(methyl methacrylate) (PMMA) and one feature of this material is its affinity for water, which leads to a swelling of the fibre and an increase of refractive index, both of which contribute to an increase in the Bragg wavelength of a FBG written in the fibre ${ }^{[3]}$. This is a potentially very useful property, which has possible applications in chemical processing, agriculture, food storage, paper manufacturing, semiconductor and pharmaceutical

*w.zhang@aston.ac.uk

21st International Conference on Optical Fiber Sensors, edited by Wojtek J. Bock, Jacques Albert, Xiaoyi Bao, Proc. of SPIE Vol. 7753, 77533M · C 2011 SPIE · CCC code: 0277-786X/11/\$18 · doi: 10.1117/12.885936 
industries, where humidity is monitored and controlled to ensure product quality. POF Bragg gratings (POFBGs) have recently been applied, for example, to quantifying the small amount of water present in aviation fuel ${ }^{[4]}$.

When POFBGs are applied to humidity sensing, a key issue is the response time, which is particularly important for the applications where accurate humidity control is required. In this work we investigate different polymer fibres and different fibre geometries. A much improved time response is achieved.

\section{EXPERIMENTS AND RESULTS}

In the reported optical fibre humidity sensors (including POFBGs and polymer coated silica FBGs) the response time ranges from $25 \mathrm{~min}$ (coating thickness of $33 \mu \mathrm{m}$ ) ${ }^{[5]}$ to $80 \mathrm{~min}$ (coating thickness of $29 \mu \mathrm{m}$ ) ${ }^{[6]}$. In reference [4] a response time of 30 min was reported when using POFBG with a diameter of $195 \mu \mathrm{m}$ while a response time of one hour was measured for a POFBG with a diameter of $125 \mu \mathrm{m}$ in [3]. Since the swelling of polymer optical fibre dominates the wavelength change of the POFBG the main influence on the humidity time response comes from the diffusion coefficient for water into the PMMA and the geometry of the POF.

We therefore carried out a series of experiments to investigate this influence on humidity time response of the diameter of POFBG humidity sensors by measuring the response time for Bragg gratings written in different polymer optical fibres with different diameters.

POFBGs were fabricated by attaching a $10 \mathrm{~cm}$ length of POF to a single mode silica fibre down-lead using UV curable glue (Norland 76). The PMMA based POF contained a $5 \mathrm{~mm}$ long FBG, fabricated by illuminating from above a phase mask of period $1.057 \mu \mathrm{m}$ placed on top of the POF using $325 \mathrm{~nm}$ light from a $\mathrm{HeCd}$ laser. For testing, the POFBG sensors were placed inside an environmental chamber (Sanyo Gallenkamp). They were illuminated with light from a broadband light source and observed in reflection using an IBSEN I-MON 400 wavelength interrogation system. Three different POF based FBGs were placed in the chamber for testing. The measured humidity responses are shown in Fig. 1 where the relative humidity (RH) was step-changed from $80 \%$ to $70 \%$ while the chamber temperature was kept at $25{ }^{\circ} \mathrm{C}$. To simplify comparison of the sensors which have slightly different Bragg wavelengths, we chose to plot the time response of the relative wavelength change, $\Delta \lambda / \Delta \lambda_{\max }$, which is the ratio of the grating's Bragg wavelength deviation from its original value to the maximum wavelength deviation induced by the relative humidity change. The diameters of POF1, POF2 and POF3 were originally $150 \mu \mathrm{m}, 190 \mu \mathrm{m}$ and $190 \mu \mathrm{m}$, respectively.

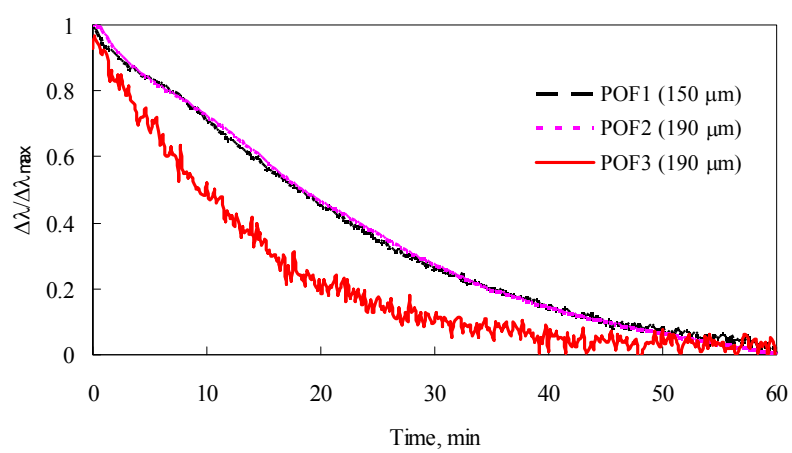

Fig.1 RH sensing time responses of POFBGs

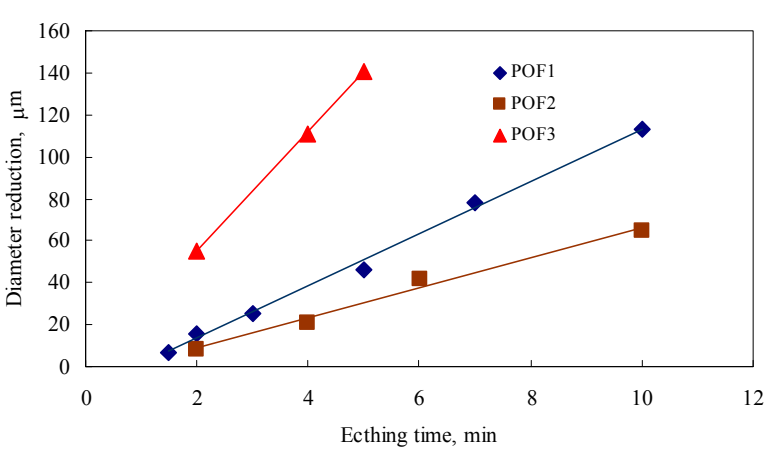

Fig. 2 POF diameter reduction due to acetone etching 
From Fig. 1 one can notice that for POF1 and POF2 the relative Bragg wavelength change is not stabilised 1 hour after the relative humidity step change. The response time then was estimated as the time of relative wavelength change being reduced to $10 \%$, with the help of an exponential fit to the experimental data. Therefore the response times for POF1, POF2 and POF3 were estimated as $58 \mathrm{~min}, 62 \mathrm{~min}$ and $38 \mathrm{~min}$, respectively.

PMMA can be easily dissolved in some chemical solvents, for example, acetone. This provides a simple and effective way to reduce the diameter of POF to a desired value. Acetone etching was applied to the aforementioned POFs, with the resulting diameter reductions being shown in Fig. 2. From Fig. 2 one can notice that POF3 shows the fastest etching rate and POF1 the slowest. Considering the results shown in Fig. 1 there seems to be a possible link between the response time and etching rate of the POF. POF3 shows the fastest etching rate and shortest response time and POF1 shows the slowest etching rate and a long response time though the fibre diameter is much smaller than the other two. It seems that the high diffusion rate of water that leads to a short humidity response rate may be correlated with a fast diffusion of acetone into the PMMA permitting more rapid etching. We are currently investigating the roles played by the PMMA molecular weight distribution and the fibre drawing process in this effect.

We then etched several Bragg gratings written in POF2 and POF3. The etched POFBGs were placed in the environmental chamber to enable investigation of the time response to relative humidity. Fig. 3 shows some typical results when the environmental chamber was set to the same condition as used previously (RH step change from $80 \%$ to $70 \%$, T set at $25{ }^{\circ} \mathrm{C}$ ). Again the response time for the FBG's relative wavelength change to be reduced to $10 \%$ was estimated. All the response times are summarized in Table 1.

Table 1. RH sensing response times of different POFs

\begin{tabular}{cc}
\hline POF FBG & Response time \\
\hline POF1 $(150 \mu \mathrm{m})$ & $58 \mathrm{~min}$ \\
POF2 $(190 \mu \mathrm{m})$ & $62 \mathrm{~min}$ \\
POF2 $(153 \mu \mathrm{m})$ & $58 \mathrm{~min}$ \\
POF2 $(131 \mu \mathrm{m})$ & $39 \mathrm{~min}$ \\
POF3 $(190 \mu \mathrm{m})$ & $38 \mathrm{~min}$ \\
POF3 $(135 \mu \mathrm{m})$ & $11 \mathrm{~min}$ \\
\hline
\end{tabular}

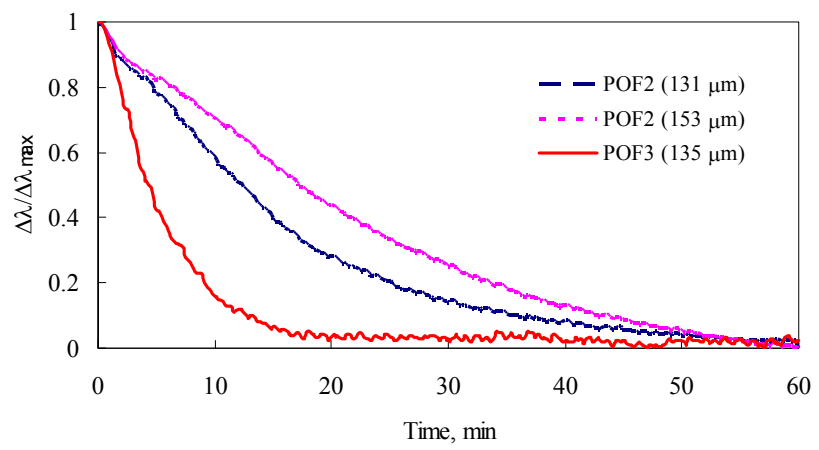

Fig. 3 RH sensing time responses of etched POFBGs

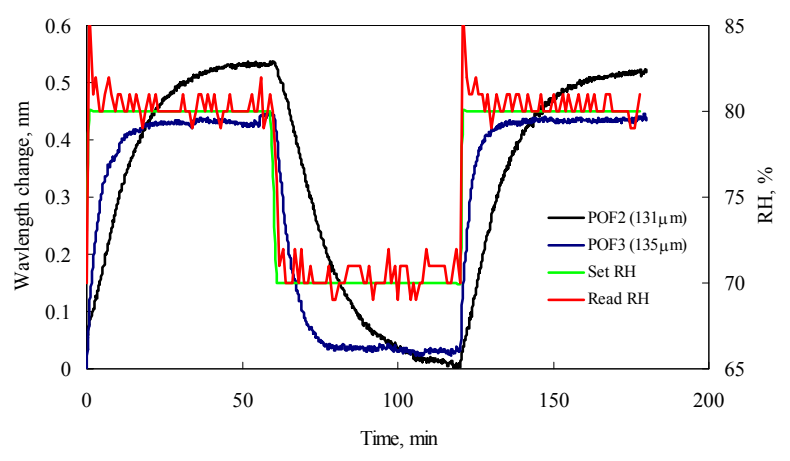

Fig. 4 Full cycle responses to RH step change 
The results listed in the table show a clear trend that for the same type of POF the response time for humidity sensing decreases with reduced fibre diameter. POF3 shows the best performance among the POFs used in this work in which the response time is greatly reduced to $11 \mathrm{~min}$. This is a satisfying speed for applications such as water in fuel sensing. Further reducing the diameter in POF3 could provide shorter response time; however, it may degrade the grating reflection and compromise the ease of handling.

Fig. 4 shows the full cycle response for humidity sensing using POF2 $(131 \mu \mathrm{m})$ and POF3 $(135 \mu \mathrm{m})$ where RH varied from $80 \%$ to $70 \%$ and back to $80 \%$. As a comparison the set value and measured value from the environmental chamber's built-in RH sensor are also depicted in the figure.

\section{CONCLUSION}

We have experimentally investigated the influence of different polymer optical fibres with different diameters on the time response of humidity sensing. Controllable etching has been used to reduce the fibre diameter. A much improved humidity sensing response time has been achieved by using a POFBG with etching reduced diameter. Based on this technique the response of POFBG humidity sensors can be improved to enhance performance for many potential applications.

\section{REFERENCES}

[1] Xiong, Z., Peng, G.D., Wu, B., and Chu, P.L., "Highly tunable Bragg gratings in single-mode polymer optical fibers," IEEE Photon. Tech. Lett., 11(3), 352-354 (1999)

[2] Dobb, H., Webb, D. J., Kalli, K., Argyros, A., Large,M. C. J., and van Eijkelenborg, M. A., " Continuous wave ultraviolet light-induced fiber Bragg gratings in few- and single-mode microstructured polymer optical fibers," Opt. Lett., 30(24), 3296-3298 (2005)

[3] Harbach, N. G., "Fibre bragg gratings in polymer optical fibres." PhD thesis Lausanne: EPFL, 2008

[4] Zhang, C, Chen, X., Webb, D. J. and Peng, G.-D., "Water detection in jet fuel using a polymer optical fibre Bragg grating", Postdeadline paper, 20th International Conference on Optical Fibre Sensors, (2009).

[5] Yeo, T.L., Sun, T., Grattan, K. T. V., Parry, D., Lade, R., Powell, B. D., "Polymer-coated fibre Bragg grating for relative humidity sensing," IEEE Sensors J., 5(5) 1082-1089 (2005)

[6] Giaccari, P., Limberger, H. G., and Kronenberg, P., "Influence of humidity and temperature on polyimide-coated fibre Bragg gratings," Proc. Bragg Gratings, Photosensitivity, and Poling in Glass fibres and Waveguides: Applications and Fundamentals, page BFB2 (2001). 